\title{
Prekarisierung der Arbeit - Prekarisierung im Lebenszusammenhang
}

Ausgangspunkt der Debatte um „Prekarisierung“ im letzten Jahrzehnt war die Ausbreitung unsicherer Beschäftigungsverhältnisse. Die Zahl der abhängig Beschäftigten, die kein reguläres Normalarbeitsverhältnis haben, sondern mit befristeter Beschäftigung, Leiharbeit, Mini-Jobs, unfreiwilliger Teilzeitarbeit oder Soloselbstständigkeit ihren Lebensunterhalt erwerben, hat stetig zugenommen. Verbreitet haben sich damit Beschäftigungsformen, die nur ein geringes Maß an sozialer Sicherheit aufweisen, die den Betroffenen soziale Teilhabe und Zukunftsgestaltung erschweren. Daran hat auch die Aktivierungspolitik der letzten Jahre Anteil, weil soziale Sicherungsniveaus wie auch die wohlfahrtsstaatliche Verantwortung insgesamt reduziert worden sind.

Pierre Bourdieu hatte bereits im vergangenen Jahrhundert auf die Machtdimension von Prekarität hingewiesen. Sie sei eine Herrschaftsform. Denn die Unsicherheit wirkt letztlich disziplinierend. Bisher ist allerdings kaum näher ausgeleuchtet worden, wie diese Disziplinierung erfolgt und welches Disziplinarregime als Begleiterscheinung prekärer Erwerbsarbeit und Lebensverhältnisse greift.

Der Diskurs um Prekarisierungsprozesse hat einen zeitdiagnostischen und gesellschaftskritischen Anspruch. Der soziale Wandel kann indes unterschiedlich gedeutet werden. Auch der Begriff der „Entgrenzung“ thematisiert die Erosion des Normalarbeitsverhältnisses und Auflösung bisheriger Grenzziehungen. Er nimmt aber stärker die widersprüchlichen und auch positiven Seiten, die mit den Deregulierungs- und Flexibilisierungsprozessen einhergehen, in den Blick.

Bereits vor Jahren hat die feministische Forschung den Prekaritätsdiskurs als verkürzt und einseitig kritisiert. Er sei zum einen geschlechtsblind, weil der Verlust von Standards der Beschäftigung(ssicherheit) thematisiert wird, die niemals für alle, vor allem nicht für weibliche Beschäftigte gegolten haben. Zum anderen nähme er einseitig die Erwerbsarbeit in den Blick, ohne die gesellschaftlich notwendige Fürsorgearbeit (Care) für Kinder und Pflegebedürftige und ihre wechselseitigen Zusammenhänge mit der Erwerbsarbeit zu berücksichtigen. Schließlich sei zu einseitig nur der Verlust von Sicherheiten betont worden, ohne dass nach dem entstehenden Neuen gefragt würde. Im Zuge der Erosion des Normalarbeitsverhältnisses, das letztlich auf traditionellen Formen der geschlechtlichen Arbeitsteilung basiert, könnten auch neue Arbeits- und Lebensformen entstehen. Aus dieser Kritik entwickelte sich eine fruchtbare Suche nach erweiterten Konzepten, um die gegenwärtigen Prozesse adäquat zu erfassen. Von unterschiedlichen Perspektiven aus sind begriffliche Erweiterungen von „Prekarität“ und „Prekarisierung“ entwickelt worden.

Demnach reicht es erstens nicht aus, ausschließlich Beschäftigungsverhältnisse zu betrachten, sondern es geht auch um die
Qualität der Erwerbsarbeit. Für die Einlösung des mit Erwerbsarbeit verbundenen Versprechens sozialer Teilhabe sind nicht allein die Sicherheit des Arbeitsplatzes, das Einkommen und das Niveau sozialer Sicherung ausschlaggebend. Vielmehr geht es auch um förderliche Arbeitsinhalte, den Erhalt der Beschäftigungsfähigkeit sowie Möglichkeiten der Interessenwahrnehmung. Einige Autorinnen und Autoren identifizieren Formen von Prekarität deswegen auch innerhalb scheinbarer Normalarbeit, wenn etwa extreme Arbeitsbelastung, Verletzung von Rechten und menschlicher Würde zum Arbeitsalltag gehören.

Zweitens muss Prekarität in den lebensweltlichen Kontext eingeordnet werden. Atypische Beschäftigung ist unter Umständen nur potenziell prekär: Erst im Haushaltszusammenhang entscheidet sich, welche Folgen entsicherte und entgrenzte Erwerbsarbeit auf den Lebenszusammenhang der Einzelnen zeitigt. Auch sind die Verflechtungen zwischen Erwerbsarbeit und anderen Lebensbereichen in zeitlicher, räumlicher u.a. Hinsicht zu beachten. D.h. es geht bei der Analyse der Lebensführung nicht um die Auswirkungen prekärer Erwerbsarbeit allein, sondern in den Blick geraten muss auch, dass die Entgrenzung von Familie und Geschlechterverhältnissen eigene Dynamiken und Widersprüchlichkeiten in die Lebensverhältnisse bringt.

Drittens sind die Interaktionen der Haushaltsmitglieder in die Analyse mit einzubeziehen. Die Menschen sind den Umwälzungen nicht hilflos ausgeliefert. Vielmehr mobilisieren sie Ressourcen im Umgang mit Prekarität. Unter Umständen werden Geschlechterarrangements neu verhandelt und neue Umgangsweisen mit Instabilität entwickelt.

Viertens geht es um die biografische Perspektive. Nur im Verlauf der (Erwerbs-)Biografie wird erkennbar, welche Bedeutung (potenziell) prekäre Beschäftigungsverhältnisse für die Betroffenen haben.

Die Beiträge in diesem Heft behandeln solche bislang wenig beachteten Dimensionen von Prekarität und schlagen ein erweitertes Prekaritätsverständnis vor. Welche Konsequenzen Prekarisierungsprozesse für die individuelle und kollektive Handlungsfähigkeit haben, bleibt zu diskutieren. Gewerkschaften und Selbstorganisationsformen von Prekarisierten haben je eigene Antworten darauf. 